\title{
Echocardiographic manifestations of COVID -19 infection
}

\author{
Rahul Vasudev ${ }^{1}$, Nirmal Guragai ${ }^{1}$, Habib Habib ${ }^{1}$, Kevin Hosein ${ }^{1}$, Hartaj Virk ${ }^{2}$, Irvin \\ Goldfarb $^{3}$, Raja Pullatt ${ }^{3}$, Mahesh Bikkina ${ }^{1}$, and Fayez Shamoon ${ }^{1}$ \\ ${ }^{1}$ St Josephs Reg Med Ctr \\ ${ }^{2}$ Seton Hall University \\ ${ }^{3}$ Affiliation not available
}

April 28, 2020

\begin{abstract}
Introduction: The cardiovascular complications related to COVID-19 have led to the need for echocardiographic services for these patients to help with the management and identification of complications. The aim of this study was to identify echocardiographic findings in COVID-19 infection and its utility in management. Methods: We included in our study patients who were COVID-19 positive by polymerase chain reaction test (PCR) and had echocardiogram done during the same admission. Results: A total of 45 patients were evaluated, the mean age was $61.4 \pm 12.2$ years. Hypertension 29 (64\%) and in diabetes mellitus in 25 patients ( $55 \%$ ) were the most common comorbidities followed by congestive heart failure(CHF) in 11 patients (24\%), Coronary artery disease (CAD) in 9 patients (20\%) and valvular heart disease in 3 patients $(7 \%)$. Eight patients $(18 \%)$ had of myocardial injury suggested by elevated troponin $(\mathrm{n}=44)$ and BNP was found to be elevated in 14 patients (35.8\%) $(\mathrm{n}=39)$ patients. Majority $93 \%(\mathrm{n}=29)$ had elevated LDH and $87.8 \%(\mathrm{n}=33)$ had elevated ferritin. and D dimer $88 \%(\mathrm{n}=27)$. 14 patients had left ventricular dysfunction (31\%). 6 patients with right ventricular (RV) dilatation and 5 patients with reduced RVEF. Pressure and volume overload were seen in 3 of our patients. In one patient RV thrombus was visualized. Pulmonary pressure was found to be elevated in 10 patients (24\%). Conclusion: 2-dimensional echocardiography can be an important bedside tool in the assessment of left and right ventricular function and hemodynamic status COVID-19 patients.
\end{abstract}

\section{Introduction:}

After its emergence in Wuhan, Hubei province of China in December 2019 novel Corona virus disease (COVID-19) infection has grown into one of the largest healthcare crises of the last century. Early reports from China and later Europe indicated that the angiotensin converting enzyme 2 (ACE 2) surface protein acts as a receptor for this virus and is the port of entry for the virus into the cell. These receptors are also expressed in myocardium, reflecting the link between heart and the virus. Emerging data has shown that this novel virus affects the cardiovascular system in several ways leading to increased morbidity and mortality. Several case reports and recently published epidemiological data have shown that patients with myocardial involvement and COVID -19 infection have poor outcomes [1]. The other described effects of this viral infection on the cardiovascular system include myocardial infarction, acute exacerbation of heart failure, arrhythmias and procoagulant effects. The cardiovascular complications related to COVID-19 have led to the need of echocardiographic services for these patients to help with the management and identification of complications. The aim of this study was to identify echocardiographic findings in COVID-19 infection and its utility in management .

\section{Methods:}

The study was conducted between March 15, 2020 to April 15, 2020 during the COVID-19 pandemic in three different hospitals in Northern New Jersey, USA, which continues to be one of the COVID-19 hotspots in the United states. We included in our study patients who were COVID positive by polymerase chain 
reaction test $(\mathrm{PCR})$ and had echocardiogram done during the same admission. Due to the high risk to the sonographers for exposure, echocardiography was performed only on patients where it was expected to alter management and to identify possible complications. All of the studies were performed in patient rooms on medical floors and intensive care units to decrease the risk of spread of infection while moving patients. The equipment was cleaned pre and post procedures as per guidelines set up by the infectious disease control of the hospital. All echocardiographic parameters were obtained as recommended by American Society of echocardiography (ASE). The echocardiographic findings were interpreted by a board certified cardiologist. Historical and laboratory data was obtained retrospectively from electronic medical record review.

\section{Results}

A total of 45 patients were included in the study, the mean age of these patients was $61.4 \pm 12.2$ years (range 28-81). Males and females were equally represented in our data $(\mathrm{M}=23, \mathrm{~F}=22)$. Hispanic race constituted $47 \%$ of the patient population $(\mathrm{n}=21)$ followed by African Americans $27 \%(\mathrm{n}=12)$, Caucasians $20 \%(\mathrm{n}=9)$ and other races $6 \%(\mathrm{n}=3)$. Hypertension in $29(64 \%)$ and diabetes mellitus in 25 patients ( $55 \%)$ were the most common co morbidities associated with COVID-19 infection followed by congestive heart failure(CHF) in 11 patients $(24 \%)$, Coronary artery disease (CAD) in 9 patients $(20 \%)$ and valvular heart disease in 3 patients (7\%). Eight patients (18\%) had of myocardial injury suggested by elevated troponin $(\mathrm{n}=44)$ and BNP was found to be elevated in 14 patients $(35.8 \%)(\mathrm{n}=39)$ patients. Among other inflammatory biomarkers, majority $93 \%(n=29)$ had elevated LDH and $87.8 \%(n=33)$ had elevated ferritin. Similarly $88 \%(n=27)$ had elevated D-dimer suggesting that they might be in a prothrombotic state. This laboratory data and echocardiographic findings are summarized in table1.

A total of 14 patients had left ventricular dysfunction (31\%), 7 of these patients had mild dysfunction with left ventricular ejection fraction (LVEF) 41-50\%, 2 had moderate dysfunction (LVEF 30-40\%) and 5 patients had severe LV dysfunction ( $\mathrm{LVEF}<30 \%)$. LV thrombus was not seen in any patients. Regional wall motion abnormality was seen in two patients; one patients presenting with AMI and another with takotsubo cardiomyopathy. Rest of the patients with low EF had global hypokinesis. None of the patients had pericardial effusion or severe valvulopathy secondary to COVID-19 infection. We found 6 patients with right ventricular (RV) dilatation and 5 patients with reduced RVEF. Pressure and volume overload, as demonstrated by flattening of interventricular septum (IVS) in diastole and systole, was seen in 3 of our patients. In one patient RV thrombus was visualized, which was confirmed on computed tomography angiography (CTA) of the chest. Pulmonary pressure (calculated using tricuspid regurgitation jet and right atrial pressure) was found to be elevated in 10 patients $(24 \%)$ in the study population.

\section{Discussion}

The unequal distribution of race in our study likely reflects the demographics that our institute caters to. Although there have been reports that Hispanic and African American patients are disproportionately more effected and have worse outcomes. Hypertension and diabetes mellitus were the most common co morbidities in our patients associated with COVID-19 infection as seen in most of the previously published literature. Elevated brain natriuretic peptide (BNP) and cardiac troponin (cTr) have been associated with myocardial injury and poor outcomes. Inflammatory biomarkers also play an important role in risk stratification of disease severity and prognostication. The prevalence of elevated biomarkers in our study was higher than the $8-12 \%$ observed by Lippi et al [2]. This was most likely the result of selection bias of performing echocardiograms on the patients suspected to have cardiac injury. Other inflammatory biomarkers, like LDH , ferritin. and D-dimer suggesting were elevated in majority of our patients. These markers have been found to be associated with severe disease, hyper coagulation and increased mortality.

Myocarditis based on elevated troponins and inflammatory markers has been described previously [1] but no data on echocardiographic findings in these patients is available. The etiology of left ventricular dysfunction can be multifactorial in these patients. These patients can have acute myocardial infarction(AMI) due to plaque rupture secondary to stress of infection as was seen in one of our patients who had complete occlusion on the mid left anterior descending artery and underwent percutaneous intervention. One patient in our study 
group developed stress related cardiomyopathy secondary to COVID-19 infection. He presented as cardiac arrest with minimal troponin elevation, echocardiogram was typical for takotsubo cardiomyopathy. Other possible etiologies for poor LV function in these patients could be myocarditis and prior LV dysfunction. Two echocardiograms were requested to rule LV thrombus as the patients presented with acute limb ischemia but LV thrombus was not seen in any patients in our study including these two patients. The arterial thrombosis in these patients could result from coagulopathy secondary to disseminated intravascular coagulation, heparininduced thrombocytopenia, thrombotic microangiopathy and antiphospholipid antibodies [3].

We had 2 patients with pulmonary embolism (PE) in our study group, echocardiogram guided diagnosis in these patients. There is a very high risk of thromboembolism including pulmonary embolism in patients with severe COVID-19 infection. Recently published data suggests incidence of thromboembolism as high $31 \%$ critically ill patients, pulmonary embolism was the most frequent (81\%) thromboembolic complication in these patients [4]. In our study RV thrombus was visualized in one patient, which was confirmed on computed tomography angiography (CTA) of the chest, showing diffuse pulmonary embolism. She was treated with low molecular weight heparin and repeat echocardiogram done 2 weeks later showed near complete resolution of thrombus. The other patient with PE who was a 28 year old female with COVID -19 infection and no other risk factors, presented with severe shortness of breath was found to have CTA confirmed extensive bilateral pulmonary embolism after her echocardiogram showed RV dilatation with flattening of IVS. She received tissue plasminogen activator (tPA) and was eventually discharged home on apixaban. There is growing consensus that patients with COVID -19 infection have a prothrombotic state and development of microthrombi in pulmonary vasculature as demonstrated by pathological studies [5], this most likely could explain the elevated pulmonary pressures and RV dysfunction in these patients. Emerging evidence suggests that anticoagulation could help a selected group of patients[6], echocardiogram may act as an aid to identify these patients. Also hypoxemia and high positive end expiratory pressure ventilation requirement in these patients can lead to RV dysfunction and eventually poor cardiac output. Bedside echocardiogram in critical care units can help in identifying features of RV dysfunction and help in management of ventilators to minimize adverse effects on cardiac output.

In conclusion, 2-dimensional echocardiography can be an important bedside tool in assessment of left and right ventricular function and hemodynamic status COVID-19 patients. When appropriately chosen for the correct group of patients echocardiogram can help in navigating management options and identifying complications.

Table 1 showing echocardiographic and laboratory parameters

\begin{tabular}{ll}
\hline Echocardiography Findings $(n=45)$ & Number of patients \\
\hline Left ventricular ejection fraction decreased & 14 \\
Regional wall motion abnormality & 2 \\
Takotsubo Cardiomyopathy & 1 \\
LV thrombus, pericardial effusion & 0 \\
Right Ventricular dilatation & 6 \\
Right Ventricular ejection fraction decreased & 5 \\
Pressure and Volume Overload & 3 \\
Right Ventricle Thrombus & 1 \\
Pulmonary Hypertension & 10 \\
Laboratory findings $(\mathrm{n}=$ total test available) & Laboratory findings $(\mathrm{n}=$ total test available) \\
Elevated BNP $(\mathrm{n}=39)$ & 14 \\
Elevated troponin $(\mathrm{n}=44)$ & 8 \\
Elevated Lactate dehydrogenase $(\mathrm{n}=29)$ & 27 \\
Elevated Ferritin $(\mathrm{n}=33)$ & 29 \\
Elevated d-dimer $(\mathrm{n}=27)$ & 24 \\
\hline
\end{tabular}




\section{References}

1. Shi S, Qin M, Shen B, et al. Association of Cardiac Injury With Mortality in Hospitalized Patients With

COVID-19 in Wuhan, China.JAMA Cardiol. Published online March 25, 2020. doi:10.1001/jamacardio.2020.0950

Lippi G., Plebani M. Laboratory abnormalities in patients with COVID-2019 infection. Clin Chem Lab Med. 2020.

1. Y. Zhang, M. Xiao, S. Zhang, P. Xia, W. Cao, W. Jiang, H. Chen, X. Ding, H. Zhao, H. Zhang, C. Wang, J. Zhao, X. Sun, R. Tian, W. Wu, D. Wu, J. Ma, Y. Chen, D. Zhang, J. Xie, X. Yan, X. Zhou, Z. Liu, J. Wang, B. Du, Y. Qin, P. Gao, X. Qin, Y. Xu, W. Zhang, T. Li, F. Zhang, Y. Zhao, Y. Li, S. Zhang, Coagulopathy and Antiphospholipid Antibodies in Patients with Covid -19, N. Engl. J. Med. (2020). https://doi.org/10.1056/NEJMc2007575

2. F.A. Klok, et al., Thrombosis Research, https://doi.org/10.1016/j.thromres.2020.04.013

3. Fox, Sharon \& Akmatbekov, Aibek \& Harbert, Jack \& Li, Guang \& Brown, J. \& Heide, Richard. (2020). Pulmonary and Cardiac Pathology in Covid-19: The First Autopsy Series from New Orleans. 10.1101/2020.04.06.20050575.

4. Tang, N., Bai, H., Chen, X., Gong, J., Li, D. and Sun, Z. (2020), Anticoagulant treatment is associated with decreased mortality in severe coronavirus disease 2019 patients with coagulopathy. J Thromb Haemost. Accepted Author Manuscript. doi:10.1111/jth.14817 